STRUCTURE-ACTIVITY RELATIONSHIPS

OF ANTI-TUMOUR AGENTS 


\section{DEVELOPMENTS IN PHARMACOLOGY}

VOLUME 3

1. J.M. Boeynaems and A.G. Herman, eds.: Prostaglandins, prostacyclin, and thromboxanes measurement. 1980. ISBN 9024724171.

2. R.M. Berne, Th.W. Rall and R. Rubio, eds.: Regulatory function in adenosine. 1983. ISBN 9024727790. 


\title{
STRUCTURE-ACTIVITY RELATIONSHIPS OF ANTI-TUMOUR AGENTS
}

\author{
Edited by \\ D.N. Reinhoudt \\ Department of Organic Chemistry \\ Technical University Twente, Enschede, The Netherlands
}

T.A. Connors

MRC Toxicology Unit

Medical Research Council Laboratories

Carshalton, Surrey, Great Britain

H.M. Pinedo

Department of Biochemistry

Antonie van Leeuwenhoekziekenhuis

Amsterdam, The Netherlands

K.W. van de Poll

Koningin Wilhelmina Fonds

The Netherlands Cancer Foundation

Amsterdam, The Netherlands

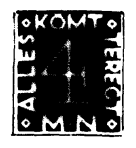

1983

MARTINUS NIJHOFF PUBLISHERS

THE HAGUE / BOSTON / LONDON 


\section{Distributors:}

for the United States and Canada

Kluwer Boston, Inc.

190 Old Derby Street

Hingham, MA 02043

USA

for all other countries

Kluwer Academic Publishers Group

Distribution Center

P.O. Box 322

$3300 \mathrm{AH}$ Dordrecht

The Netherlands

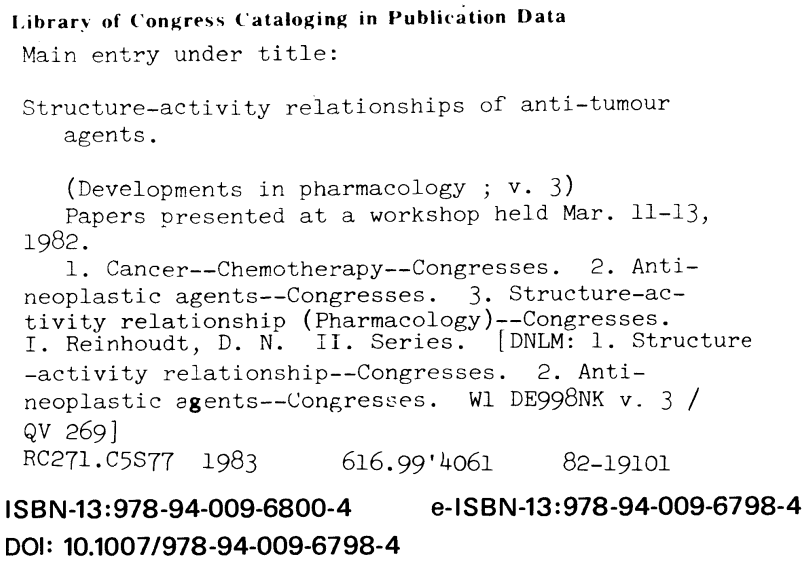

Copyright $(\uparrow) 1983$ by Martinus Nijhoff Publishers, The Hague, Boston, London. Softcover reprint of the hardcover 1st edition 1983

All rights reserved. No part of this publication may be reproduced, stored in a retrieval system, or transmitted in any form or by any means, mechanical, photocopying, recording, or otherwise, without the prior written permission of the publisher,

Martinus Nijhoff Publishers, P.O. Box 566, 2501 CN The Hague, The Netherlands. 


\section{CONTENTS}

LIST OF AUTHORS - PARTICIPANTS

VII

1. INTRODUCTION

H.M. Pinedo

2. STRATEgY FOR THE DISCOVERY AND DEVELOPMENT OF NOVEL ANTICANCER AGENTS

V.L. Narayanan

3. QUANTITATIVE STRUCTURE-ACTIVITY RELATIONSHIP STUDIES AROUND CYTOTOXIC DRUGS

R.F. Rekker

4. ALKYLATING PRODRUGS IN CANCER CHEMOTHERAPY

T.A. Connors

5. STRUCTURE-ACTIVITY RELATIONSHIPS FOR ANTI-TUMOUR PLATINUM COMPLEXES

M.J. Cleare

6. BIOREDUCTIVE ALKYLATION - NATURALLY OCCURRING QUINONES AS POTENTIAL CANDIDATES

H.W. Moore, K.F. West, K. Srinivasacher and R. Czerniak

7. STRUCTURE-ACTIVITY RELATIONSHIPS IN DOXORUBICIN RELATED COMPOUNDS

F. Arcamone

8. STRUCTURE-ACTIVITY RELATIONSHIPS AMONG ANTITUMOUR QUINONES

J.S. Driscoll

9. IN VITRO OXIDATIVE ACTIVATION: A MODEL FOR THE CYTOTOXIC ACTION OF 9-HYDROXY ELLIPTICINE DERIVATIVES

B. Meunier, C. Auclair, J. Bernadou, G. Meunier, M. Maftouh,

S. Cros, B. Monsarrat and C. Paoletti

10. DTIC: A SPRINGBOARD TO NEW ANTITUMOUR AGENTS

M.F.G. Stevens

11. NITROSOUREAS

J.A. Montgomery 
12. OXAZAPHOSPHORINE CYTOSTATICS: STRUCTURE-ACTIVITY RELATIONSHIPS, SELECTIVITY AND METABOLISM, REGIONAL DETOXIFICATION

N. Brock

13. STUDIES ON ANTITUMOUR ANTIBIOTICS, LOW MOLECULAR WEIGHT IMMUNOMODIFIERS AND THEIR ANALOGS AND DERIVATIVES

H. Umezawa 


\section{LIST OF AUTHORS - PARTICIPANTS}

ARCAMONE, F., Farmitalia Carlo Erba, Gruppo Montedison, Ricerca \& Sviluppo Chimico, Viale E. Bezzi 24, 20146 Milano, ITALY

AUCLAIR, C., Laboratoire de Biochimie-Enzymologie de l'Institut GustaveRoussy, U 147 CNRS, U 140 INSERM, 94800 Villejuif, FRANCE

BERNADOU, J., Laboratoire de Pharmacologie et Toxicologie fondamentale, CNRS, 205 Route de Narbonne, 31078 Toulouse, FRANCE

BROCK, N., Abteilung Tumorforschung der Asta-Werke Aktiengese11schaft, Postfach 140129 , D-4800 Bielefeld 14, WEST-GERMANY

CLEARE, M.J., Johnson Mattey Research Center, Blount's Court, Sonning Common, Reading RG4 9NH, GREAT-BRITAIN

CONNORS, T.A., MRC Toxicology Unit, Medical Research Council Laboratories, Woodmansterne Road, Carshalton, Surrey SM5 4EF, GREAT-BRITAIN

CROS, S., Laboratoire de Pharmacologie et Toxicologie fondamentale, CNRS, 205 Route de Narbonne, 31078 Toulouse, FRANCE

CZERNIAK, R., Department of Chemistry, University of California, Irvine, CA 92717 , U.S.A.

DRISCOLL, J.S., Acting Associate Director, Developmental Therapeutics Program, Division of Cancer Treatment, National Cancer Institute, Blair B1dg., Rm. 5A03, 8300 Colesville Road, Silver Spring, MD 20910, U.S.A.

MAFTOUH, M., Laboratoire de Pharmacologie et Toxicologie fondamentale, CNRS, 205 Route de Narbonne, 31078 Toulouse, FRANCE

MEUNIER, B., Laboratoire de Pharmacologie et Toxicologie fondamentale, CNRS, 205 Route de Narbonne, 31078 Toulouse, FRANCE

MEUNIER, G., Laboratoire de Pharmacologie et Toxicologie fondamentale, CNRS, 205 Route de Narbonne, 31078 Toulouse, FRANCE

MONSARRAT, B., Laboratoire de Pharmacologie et Toxicologie fondamentale, CNRS, 205 Route de Narbonne, 31078 Toulouse, FRANCE

MONTGOMERY, J.A., Southern Research Institute, P.O. Box 3307-A, Birmingham, AL 35255, U.S.A. 
MOORE, H.W., Department of Chemistry, University of California, Irvine, CA 92717 , U.S.A.

NARAYANAN, V.L., Chief Drug Synthesis \& Chemistry Branch, Department of Health Education \& Welfare, National Cancer Institute, Bethesda, MD 20205, U.S.A.

PAOLETTI, C., Laboratoire de Biochimie-Enzymologie de 1 'Institut GustaveRoussy, U 147 CNRS, U 140 INSERM, 94800 Villejuif, FRANCE

PINEDO, H.M., Antoni van Leeuwenhoekhuis, Afdeling Biochemie H-3, Plesmanlaan 121, 1066 CX Amsterdam, THE NETHERLANDS

POLL, K.W. van de, Koningin Wilhelmina Fonds (The Netherlands Cancer Foundation), Sophialaan 8-10, 1075 BR Amsterdam, THE NETHERLANDS

REINHOUDT, D.N., Department of Organic Chemistry, Technical University Twente, P.O. Box 217, 7500 AE Enschede, THE NETHERLANDS

REKKER, R.F., Vrije Universiteit, Vakgroep Pharmacochemie, Subfaculteit der Scheikunde, De Boelelaan 1083, 1081 HV Amsterdam, THE NETHERLANDS

SRINIVASACHER, K., Department of Chemistry, University of California, Irvine, CA 92717 , U.S.A.

STEVENS, M.F.G., The University of Ason in Birmingham, Department of Pharmacy, Gosta Green, Birmingham B4 7ET, GREAT-BRITAIN

UMEZAWA, H., Yamanouci International Ltd., 12 Hannover Street, London WIR-9 WB, GREAT-BRITAIN

WEST, K.F., Department of Chemistry, University of California, Irvine, CA 92717 , U.S.A. 